EPJ Web of Conferences 41, 07003 (2013)

DOI: $10.1051 /$ epjconf/20134107003

C) Owned by the authors, published by EDP Sciences, 2013

\title{
Ultrafast Absorption Kinetics of NADH in Folded and Unfolded Conformations
}

\author{
Z. Heiner ${ }^{1}$, T. Roland ${ }^{2}$, J. Léonard ${ }^{2}$, S. Haacke ${ }^{2}$, G. I. Groma ${ }^{1}$ \\ ${ }^{1}$ Institute of Biophysics, Biological Research Centre, H-6701 Szeged, Hungary \\ ${ }^{2}$ Institut de Physique et Chimie des Matériaux de Strasbourg, University of Strasbourg - CNRS, \\ F-67034 Strasbourg, France
}

\begin{abstract}
The non-radiative energy transfer is shown to occur on a $\sim 3 p$ s time scale for $\mathrm{NADH}$ in the folded form in $\mathrm{H}_{2} \mathrm{O}$. Addition of methanol thermodynamically favours the open form, for which energy transfer does not occur.
\end{abstract}

\section{Introduction}

Nicotinamide adenine dinucleotide (NAD + ) and its phosphorylated and reduced forms, NADP+, $\mathrm{NADH}$ and NADPH, have central roles in cellular metabolism and energy production as hydrideacceptor and hydride-donor coenzymes. Intracellular NADH exists in two forms with different fluorescence spectra and lifetimes: one is free and the other is bound to various proteins. The ratio of the two forms depends on the microenvironment and can indicate the metabolic state of a cell, providing a route for cancer diagnostic purpose (or neurodegenerative diseases, diabetes, and aging.) [1]. Time- and wavelength-resolved fluorescence spectroscopy combined with imaging (e.g. FLIM, FRET) has been widely used to extract free and protein bound NADH signals from cellular fluorescence.

NADH contains two chromophores: adenine (A) and dihydronicotinamide (NA) with absorption maxima at 260 and $340 \mathrm{~nm}$, respectively [2]. Solvents, temperature, etc. have a strong influence on the conformational structure of NADH [3,4]. Efficient fluorescence resonance energy transfer (FRET) between the two chromophores after excitation occurs only in the folded conformational state [2]. The presence of methanol $(\mathrm{MeOH})$ results in an unfolding of the NADH molecule and a decreased FRET between the chromophores.

In contrast to the detailed studies on NADH and its derivatives by picosecond fluorescence spectroscopy, only very little is known about the time scale of FRET in NADH and how it is modulated by the solvent. Here we characterize the transient absorption of $\mathrm{NADH}$ in $\mathrm{H}_{2} \mathrm{O} / \mathrm{MeOH}$ mixtures, as a function of $\mathrm{MeOH}$ content, and following the excitation of the adenine chromophore with 120 fs time resolution.

\section{Materials and methods}

A $3.0 \times 10^{-4} \mathrm{M}$ NADH solution was prepared freshly in $0.1 \mathrm{M}$ PIPES buffer and was kept at $24^{\circ} \mathrm{C}$ during the measurements. This buffer was proven optimal in avoiding thermal NADH degradation [4]. The change in the ground state absorption spectra was found negligible in the course of the absorption kinetic measurements.

This is an Open Access article distributed under the terms of the Creative Commons Attribution License 2.0, which permits unrestricted use, distribution, and reproduction in any medium, provided the original work is properly cited. 
The fraction of NADH in the folded and unfolded conformations was characterized by excitation energy transfer measurements. Fluorescence excitation spectra were taken between 240 and $440 \mathrm{~nm}$, and the energy transfer efficiency was determined by the ratio of the fluorescence intensities at 260 and $340 \mathrm{~nm}$. The relative probability of energy transfer is maximal at low $(0-30 \%) \mathrm{MeOH}$ concentration and minimal at high $\mathrm{MeOH}$ concentration [2].

Femtosecond absorption kinetics measurements were carried out by the standard pump-probe method. For the excitation of the adenine group pump pulses of $266 \mathrm{~nm}$ were generated by the third harmonic of an amplified Ti:Sapphire system $(5 \mathrm{kHz}, 40 \mathrm{fs}, 500 \mu \mathrm{J})$. It was found that at an average power of $650 \mu \mathrm{W}$ the pump did not cause any degradation of the sample $(\mathrm{OD}=0.25)$ circulating through a $0.5-\mathrm{mm}$ square-aperture quartz cell. The $330-630-\mathrm{nm}$ portion of a white light continuum was used as probe, passing through a monochromator and detected by a cooled CCD camera. The time resolution of the measurement was $\sim 120$ fs. Data are corrected for the solvent response, composed of pump-probe two-photon absorption and solvated electron signals. Noise reduction involved filtering using SVD followed by a sliding window averaging.

\section{Results and Discussion}

The time evolution of differential absorption spectra upon excitation of the A group in the absence and presence of methanol is shown in Figure 1. In both cases the signal is dominated by an increased absorption at around $350-450 \mathrm{~nm}$. This excited state absorption (ESA), falls in the range of the absorption peak of the NA group, and overcomes the related ground state bleach. As shown below, this band is due to ESA from both A and NA, with the early A-related ESA spectrum being slightly larger. An additional ESA is observed for $\lambda>500 \mathrm{~nm}$, attributed to the A group [5]. It decays very
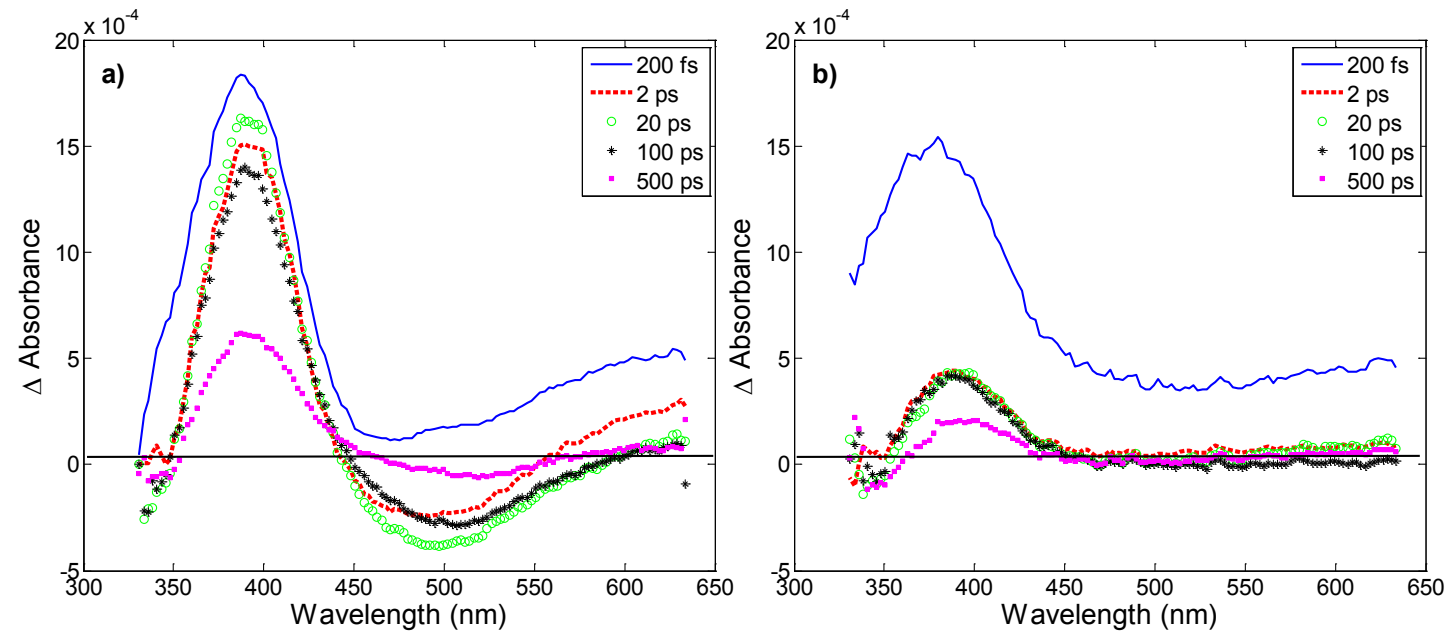

Fig. 1. The temporal changes of differential absorption spectra of NADH in $0 \%$ (a) and $80 \%$ (b) methanol.

rapidly, although somewhat slower in pure $\mathrm{H}_{2} \mathrm{O}$. Concomitant to this red-ESA decay, a negative band rises in the 450-570 $\mathrm{nm}$ region, best seen in pure $\mathrm{H}_{2} \mathrm{O}$. The maximum of the negative peak coincides well with the emission maximum of NADH $(480 \mathrm{~nm})$, hence it is interpreted as stimulated emission. At $200 \mathrm{fs}$ after the excitation the shape of the spectra are fairly similar in the two solutions. In contrast to this between 2 and 100 ps the methanol-free sample shows a pronounced change, especially in its negative peak.

The kinetics at 380 and $520 \mathrm{~nm}$ (Figure 2a and 2b) are markedly different in the two cases, in $80 \%$ methanol it decays in a few ps, while in water a second increasing phase occurs in the $10 \mathrm{ps}$ 

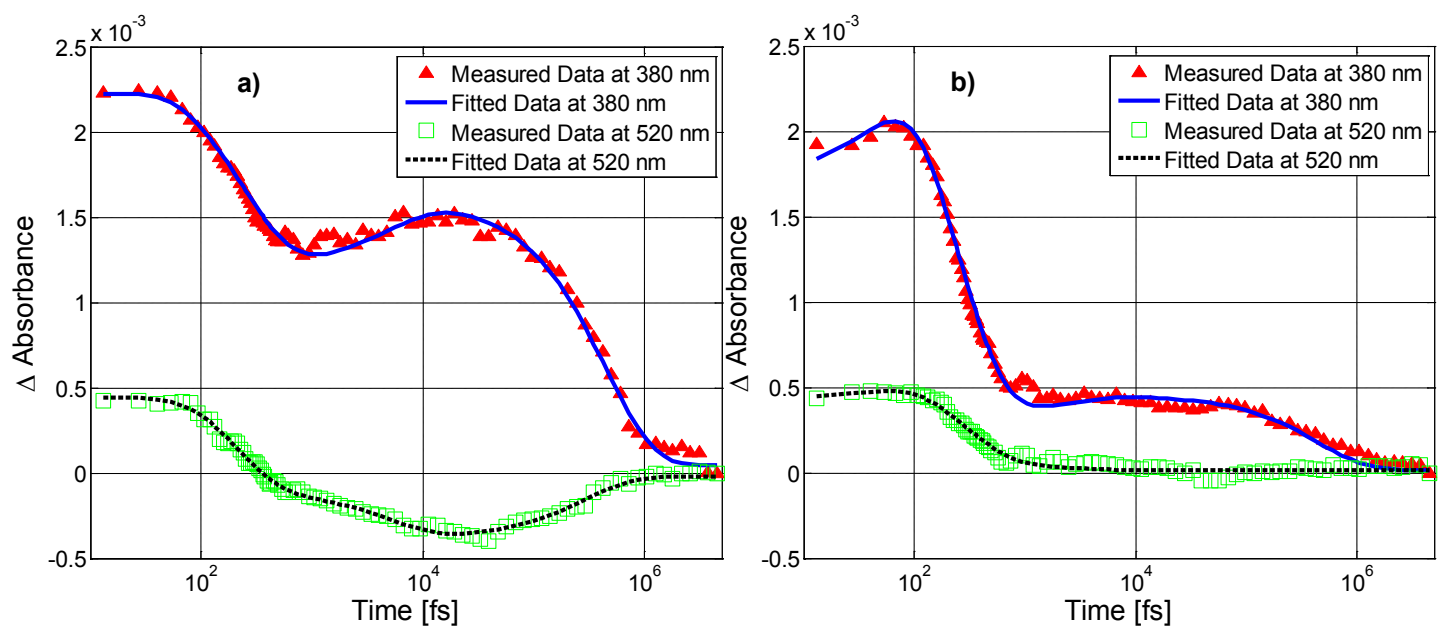

Fig. 2. Time evolution of the transient absorption of NADH in $0 \%$ (a) and $80 \%$ (b) methanol.

range. This difference is well manifested also in the time evolution of the spectra (Figure 1). It was found that the kinetics at every wavelength and methanol concentrations can be fitted by three exponentials. The fastest one with time constant of $\sim 250 \mathrm{fs}$ appears both in the presence and in the absence of methanol and can be attributed to the excited-state absorption of the A group, as we are directly exciting A. It characterizes the A-related red-ESA decay when probed at $>600 \mathrm{~nm}$ (not shown). A second and a third component of $\sim 3$ ps and $\sim 350 \mathrm{ps}$ lifetimes take place with high weight at both $380 \mathrm{~nm}$ and $520 \mathrm{~nm}$ in the methanol-free sample. The $\sim 3$ ps component is also in the redESA decay. These observations indicate that in pure water an energy transfer takes place from A to NA with the 3 ps time constant, followed by the decay of the fluorescent excited-state of NA within 350 ps. In the presence of $\mathrm{MeOH}$, the NA related long-lived ESA and SE rise with the same 3 ps time constant, but with much lower amplitudes, in line with the energy transfer being reduced. In summary, the decay of the excited state of A is biphasic, and only the slower ( $3 \mathrm{ps})$ component leads to the excitation of NA. This is possibly only if at least two ground state conformations, the open and closed forms of the NADH molecules, coexist in pure $\mathrm{H}_{2} \mathrm{O}$. Upon addition of $\mathrm{MeOH}$, the open form is thermodynamically favored, leading to the reduction of FRET.

\section{Acknowledgement}

This work was supported by National Development Agency of Hungary (TÁMOP-4.2.1, TÁMOP4.2.2, TECH-09) and co-financed by the European Regional Fund. The Strasbourg group acknowledges technical assistance from O. Crégut.

\section{References}

1. J. McGinty, N.P. Galletly, C. Dunsby, I. Munro, D.S. Elson, J. Requejo-Isidro, P. Cohen, R. Ahmad, A. Forsyth, A.V. Thillainayagam, M.A.A. Neil, P.M.W. French, G.W. Stamp, Biomed.Opt.Exp. 1, 627 (2010)

2. R.V. Hull, P.S. Conger, R.J. Hoobler, Biophys. Chem. 90, 9 (2001)

3. A.S. Ladokhin, L. Brand, J. Fluorescence, 5, 99 (1995)

4. L.R. Julio jr., C.B. Fernandes, G. de Oliveira Neto, L.T. Kubota, E. Katekawa, S. H. P. Serrano, Anal. Biochem. 260, 50 (1998)

5. B. Cohen, P.M. Hare, B. Kohler, J. Am. Chem. Soc. 125, 13594-13601 (2003). 